\title{
IS DOSE TITRATION REQUIRED FOR ANTIHYPERTENSIVE AGENTS IN GERIATRIC DIABETIC PATIENTS?
}

\author{
RAJESHWARI SHASTRY ${ }^{1 *}$, PRABHA M R ADHIKARI ${ }^{2}$, SHEETAL D ULLAL ${ }^{1}$, MUKTA N. CHOWTA ${ }^{1}$ \\ SAHANA D ACHARYA ${ }^{1}$
}

${ }^{1}$ Department of Pharmacology, Kasturba Medical College, Mangalore, Manipal Academy of Higher Education, Manipal, Karnataka, India. ${ }^{2}$ Department of Medicine, Yenepoya Medical College, Deralakatte, Yenepoya University, India. Email: rajeshwari.shastry@manipal.edu

Received: 17 August 2018, Revised and Accepted: 17 September 2018

ABSTRACT

Objective: The objective of this study is to evaluate the antihypertensive drug usage and dosage differences between geriatric and non-geriatric diabetics with reference to the duration of hypertension and creatinine clearance (Crcl).

Methods: In this observational study, patients with type 2 diabetes mellitus were grouped into geriatric (age $\geq 60$ years) and non-geriatric (age <60 years). Patients' demographic data, duration of hypertension, drugs prescribed, and serum creatinine were recorded after the patients had a stabilized antihypertensive dose for 6 months. Crcl was calculated using Cockcroft-Gault formula. The dosages of antihypertensives were converted into equivalent doses for easy comparison within a group. For angiotensin-converting enzyme inhibitors (ACEIs), enalapril was considered as prototype, and for angiotensin receptor blockers (ARBs) losartan, beta-blocker atenolol, and calcium channel blockers (CCBs), amlodipine was considered as prototype. Univariate analysis was done for comparison of drug doses between groups.

Results: A total of 336 diabetics with hypertension were included, of which 252 were geriatric and 84 non-geriatric. Duration of hypertension was expectedly longer in the geriatric group $(8.40 \pm 7.26$ vs. $5.46 \pm 5.67$; $p=0.001)$. Systolic blood pressure was higher in geriatrics $(137.14 \pm 13.51$ vs. $133.38 \pm 12.49 ; \mathrm{p}=0.01)$. When adjusted for the duration of hypertension and Crcl, there were no significant differences in the mean converted equivalent doses of beta-blockers, CCBs, ARBs, and hydrochlorothiazide between geriatrics and non-geriatrics. However, statistically significant lower converted equivalent doses of all ACEIs were needed in geriatrics compared to non-geriatrics, when adjusted for duration of hypertension and Crcl. Enalapril required $20.57 \%$ and ramipril required $18.36 \%$ dose reduction in geriatrics compared to non-geriatrics.

Conclusion: A 20\% dosage reduction is needed for ACEIs in the elderly.

Keywords: Geriatrics, Hypertension, Non-geriatrics, Diabetes.

(C) 2018The Authors. Published by Innovare Academic Sciences Pvt Ltd. This is an open access article under the CC BY license (http://creativecommons. org/licenses/by/4. 0/) DOI: http://dx.doi.org/10.22159/ajpcr.2018.v11i12.29181

\section{INTRODUCTION}

India, the world diabetes capital in the next 30 years, may also be the world hypertension capital [1]. Hypertension and diabetes mellitus (DM) are both important contributory factors for the growing burden of cardiovascular and chronic kidney disease [2]. Concomitant hypertension doubles total mortality and stroke risk, triples the risk of coronary heart disease, and significantly hastens the progression of microvascular complications, including diabetic nephropathy among diabetics [3]. Cardiovascular disease is the major cause of death in patients with DM. By 60 years, about two-thirds of hypertensives have isolated systolic hypertension (ISH); by 75 years, almost all hypertensives have ISH [4]. ISH is related strongly to the development of micro- and macrovascular diseases in patients with DM [5]. The incidence of hypertension increases with duration of type 2 diabetes; however, a substantial number of patients have hypertension at the time of diabetes diagnosis [6]. Observational studies have shown that the risk of diabetes complications significantly decreases with lower blood pressure, with the lowest risk in patients with systolic blood pressure (SBP) $<120 \mathrm{mmHg}$ [7]. Treatment of hypertension in DM is not always easy as confusion prevails regarding definition, target blood pressure, use of specific agents such as angiotensin-converting enzyme inhibitors (ACEIs), angiotensin receptor blockers (ARBs), thiazide diuretics, calcium channel blockers (CCBs), beta-blockers, and their adverse effects, especially in the elderly.

Age-related changes in kidney and liver function are the most important physiological changes of the human body which require adjustment of drug selection and dosage. The impact of diabetes on renal impairment worsens with increasing age [8]. The progressive decline in renal function that occurs with age may result in the slower elimination of drugs that are partially or completely cleared by kidneys, leading to toxicity and additive side effects when dosages are not adjusted [9]. Dosage also depends on the duration of hypertension; longer duration usually requires higher doses among the elderly. Hence, there is a dilemma in the selection of dosage of antihypertensives among the elderly; renal impairment forcing lower doses while longer duration and poor control necessitating higher doses.

Individually tailored therapeutic strategies are needed, which may have less stringent targets, for which the use of common drugs should be critically evaluated [10]. Evaluation of the morbidity profile among elderly people and the impact of age and chronic conditions on functional disability and psychological well-being are an essential part of the comprehensive assessment of the elderly. Such evaluations will have implications for providing better health care for the elderly.

For a majority of drugs, exact dosing guidelines for geriatric patients have not been established and most references do not specifically address the use of medications in the elderly. There is a clear need to focus greater attention on the unique needs of elderly diabetes patients and individualizing care. Hence, the present study was planned to evaluate the antihypertensive drug usage and dosage differences between geriatric and non-geriatric diabetics with reference to the duration of hypertension and Crcl. 


\section{METHODS}

A prospective observational study was conducted for 6 monthsin a tertiary care teaching hospital after obtaining approval from the Institutional Ethics Committee. A written informed consent was obtained from all participants. All diabetic patients with hypertension were included in the study and were grouped into geriatric (age $\geq 60$ years) and non-geriatric (age $<60$ years). Patients were followed up for 6 months to monitor their antihypertensive drug dosage. Patients who needed frequent drug dosage changes due to repeated acute illness or poor blood pressure control were not enrolled in the study. Drug treatment was not altered during the 6 months' follow-up period. The prescriptions of these patients were analyzed and the investigators did not interfere with the dose and their titration. Patients' demographic data, duration of hypertension, drugs prescribed, and serum creatinine were recorded. Crcl was calculated using Cockcroft-Gault formula. The dosages of antihypertensives were converted into equivalent doses for easy comparison within a group (Table 1). For ACEIs, enalapril was considered as prototype; for ARBs, losartan as prototype; for beta blockers, atenolol as prototype; and for CCBs, amlodipine as prototype [11].

\section{Statistical analysis}

Demographics, duration of disease, drug doses, and laboratory parameters were done using descriptive statistics. Students' unpaired t-test was used to compare the different parameters between the two groups. Univariate analysis was done for the comparison of drug doses of two groups adjusted for the duration of hypertension and Crcl. All the analyses were done at $5 \%$ significance.

\section{RESULTS}

A total of 336 diabetic hypertensive patients were included in the study ( 252 geriatrics and 84 non-geriatrics). Of the 252 geriatrics, 125 were males, and among the non-geriatrics, 38 were males. Demographic data and blood pressure values of both groups are given in Table 2. SBP was higher in geriatrics and diastolic blood pressure (DBP) was higher in non-geriatrics, and the differences were clinically insignificant.

Distribution of individual antihypertensive agents between geriatrics and non-geriatrics did not show any significant difference (Table 3).

As shown in Fig. 1, there was no significant difference between the groups with respect to usage of antihypertensive drug combinations. The most commonly used combination was ACE inhibitors with betablockers and/or thiazide diuretics (35.61\%).

Table 4 shows the antihypertensive doses received by patients. Doses of beta-blockers (metoprolol, atenolol) received by geriatrics were higher than non-geriatrics but not statistically significant. Doses of ACE inhibitors and hydrochlorothiazide received by geriatrics were lower than non-geriatrics but statistically insignificant. The doses of bisoprolol, nifedipine, and telmisartan were not compared between the groups as the sample size was too small, but they were considered for mean converted equivalent doses.

There were no significant differences in the mean converted equivalent doses of beta-blockers, CCBs, ARBs, and hydrochlorothiazide when adjusted for the duration of hypertension and $\mathrm{Crcl}$ in geriatrics and non-geriatrics (Table 5).

Table 6 gives a comparison of the duration of hypertension and Crcl adjusted mean doses of enalapril and ramipril which were lower in geriatrics but were not significant as the sample size was small. Statistically significant lower converted equivalent doses of all ACE inhibitors were seen in geriatrics compared to non-geriatrics, indicating that the dose of ACE inhibitor is nearly $1.5 \mathrm{mg}$ lower in geriatric population which is statistically and clinically significant.

\section{DISCUSSION}

Duration of hypertension was expectedly longer in the geriatric group. Geriatric diabetics had significantly high SBP compared to non-

\section{Table 1: Equivalency chart for antihypertensive agents}

\begin{tabular}{|c|c|}
\hline Prototype & Equivalent dosage of antihypertensive agents \\
\hline Atenolol $50 \mathrm{mg}$ & $\begin{array}{l}\text { Metoprolol } 100 \mathrm{mg} \text {; Bisoprolol } 5 \mathrm{mg} \text {; Nadolol } \\
40 \mathrm{mg}\end{array}$ \\
\hline Enalapril $5 \mathrm{mg}$ & $\begin{array}{l}\text { Ramipril } 2.5 \mathrm{mg} \text {; Captopril } 12.5 \mathrm{mg} \\
\text { Lisinopril } 10 \mathrm{mg}\end{array}$ \\
\hline Losartan $25 \mathrm{mg}$ & $\begin{array}{l}\text { Telmisartan } 40 \mathrm{mg} \text {; Irbesartan } 75 \mathrm{mg} \text {; } \\
\text { Candesartan } 8 \mathrm{mg}\end{array}$ \\
\hline Amlodipine $2.5 \mathrm{mg}$ & Nifedipine $30 \mathrm{mg}$ \\
\hline
\end{tabular}

Table 2: Comparison of demographics and blood pressure values between the groups

\begin{tabular}{llll}
\hline Characteristics & $\begin{array}{l}\text { Geriatrics } \\
\mathbf{n = 2 5 2}\end{array}$ & $\begin{array}{l}\text { Non-geriatrics } \\
\mathbf{n = 8 4}\end{array}$ & $\mathbf{p}$ value \\
\hline Age (years) & $68.80 \pm 6.01$ & $51.78 \pm 6.04$ & $0.001^{* *}$ \\
Body mass index & $24.78 \pm 3.81$ & $25.89 \pm 4.11$ & $0.003^{* *}$ \\
Duration of DM (years) & $12.04 \pm 9.08$ & $7.23 \pm 6.44$ & $0.001^{* *}$ \\
Duration of HTN (years) & $8.40 \pm 7.26$ & $5.46 \pm 5.67$ & $0.001^{* *}$ \\
Crcl (ml/min) & $57.82 \pm 17.41$ & $88.07 \pm 24.60$ & $0.001^{* *}$ \\
FBS (mg/dl) & $144.37 \pm 31.10$ & $151.13 \pm 29.77$ & 0.58 \\
HbA1c (\%) & $7.68 \pm 1.32$ & $7.84 \pm 1.35$ & 0.97 \\
SBP (mm/Hg) & $137.14 \pm 13.51$ & $133.38 \pm 12.49$ & $0.01^{* *}$ \\
DBP (mm/Hg) & $81.06 \pm 6.29$ & $82.38 \pm 5.75$ & 0.05 \\
\hline
\end{tabular}

Values are expressed as mean \pm SD ${ }^{*} p<0.05$; ${ }^{* *} \mathrm{p}<0.01$. DM: Diabetes mellitus, HTN: Hypertension, FBS: Fasting blood glucose, HbA1c: Hemoglobin A1c SBP:XSystolic blood pressure, DBP: Diastolic blood pressure

Table 3: Distribution of individual antihypertensive agents

\begin{tabular}{llll}
\hline Drugs & $\begin{array}{l}\text { Geriatrics } \\
\mathbf{n = 2 5 2} \mathbf{( \% )}\end{array}$ & $\begin{array}{l}\text { Non-geriatrics } \\
\mathbf{n = 8 4}(\mathbf{\%})\end{array}$ & p value \\
\hline Metoprolol & $46(18.25)$ & $15(17.85)$ & 0.58 \\
Atenolol & $58(23.01)$ & $22(26.19)$ & 0.66 \\
Bisoprolol & $6(2.38)$ & $2(2.38)$ & 1.00 \\
Amlodipine & $87(34.52)$ & $24(28.57)$ & 0.38 \\
Nifedipine & $3(1.19)$ & $3(3.57)$ & 0.34 \\
Losartan & $58(23.01)$ & $16(19.04)$ & 0.54 \\
Telmisartan & $6(2.38)$ & $4(4.76)$ & 0.46 \\
Ramipril & $34(13.49)$ & $11(13.09)$ & 0.93 \\
Enalapril & $67(26.58)$ & $22(26.19)$ & 0.94 \\
Hydrochlorothiazide & $35(13.88)$ & $10(11.90)$ & 0.78 \\
\hline
\end{tabular}

*Total exceeds $100 \%$, since the average patients received more than one drug

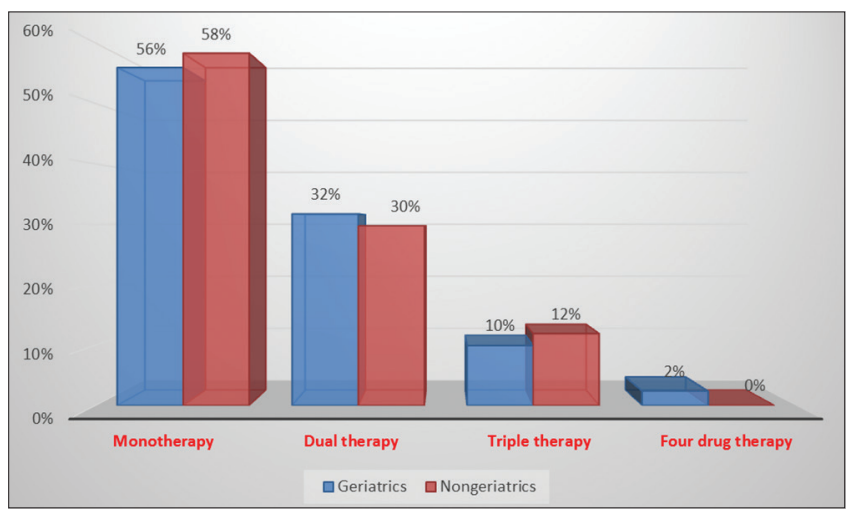

Fig. 1: Use (\%) of antihypertensive drug combinations

geriatrics, while non-geriatrics had higher diastolic blood pressure although this was clinically and statistically insignificant. This increased systolic hypertension in the elderly which occurs with aging results from stiffening of the major capacitance arteries [12].

The choice of antihypertensive agents in diabetics is based on previous medication history, comorbid conditions as well as propensity of 
drug to reduce adverse cardiovascular and metabolic events. In the present study, with respect to the choice of antihypertensive agents, there was no significant difference between geriatrics and nongeriatrics. Renin-angiotensin aldosterone system inhibitors, namely ACE inhibitors (39.9\%) and ARBs (25\%), were the most commonly used antihypertensive agents followed by beta-blockers (44.34\%). The usage of beta-blockers was more compared to CCBs/diuretics as these diabetic hypertensive patients had ischemic heart disease as one of the comorbidities. Hence, the prescribing pattern of antihypertensive agents in diabetic patients in our study follows the current JNC 8 guidelines [13]. In contrast, in another study, the most commonly used antihypertensives in geriatrics were CCB because the patient population was non-diabetics [14]. According to the literature, elderly patients require more number of antihypertensive agents compared to their younger counterparts $[15,16]$. However, in the present study, there was no significant difference in the number of antihypertensive agents used between the groups, which could be due to the reason that combination of drugs is probably prescribed in younger adults in whom a tighter control of blood pressure is intended compared to geriatrics. It is also noteworthy that a combination of four antihypertensive drugs was prescribed only for geriatric diabetics. With regard to the combination regimen, there is no clear-cut evidence to support the superiority of one combination over the others. In our study, ACE inhibitors with beta-blockers and/or thiazide diuretics were the most commonly used combination.

It is known that beta-blocker responses are blunted with age, and hence, higher doses are required in geriatrics. The dosage of betablockers needs to be adapted to GFR [8]. Metoprolol dose appeared to be higher in geriatrics (60 vs. $55 \mathrm{mg}$ ), whereas atenolol dose lower in geriatrics (42 vs. $43 \mathrm{mg}$ ) which was statistically and clinically insignificant. Hydrophilic beta-blockers (e.g., atenolol, bisoprolol, nadolol, and acebutolol) are eliminated renally and dosing adjustments are expected in patients with chronic kidney disease [17]; however, in our study, there was no reduction in the atenolol dose probably

Table 4: Comparison of doses of antihypertensive drugs

\begin{tabular}{lllllll}
\hline \multirow{2}{*}{ Drugs } & \multicolumn{2}{l}{ Geriatrics } & & \multicolumn{2}{l}{ Non-geriatrics } & \multirow{2}{*}{ p value } \\
\cline { 2 - 3 } & $\mathbf{n}$ & Doses (mg) & & $\mathbf{n}$ & Doses (mg) & \\
\hline Metoprolol & 46 & $61.41 \pm 28.72$ & & 15 & $56.66 \pm 24.02$ & 0.57 \\
Atenolol & 58 & $42.02 \pm 12.97$ & & 22 & $43.18 \pm 11.39$ & 0.71 \\
Amlodipine & 87 & $5.17 \pm 2.55$ & & 24 & $5.10 \pm 2.14$ & 0.97 \\
Losartan & 58 & $40.73 \pm 16.97$ & & 16 & $45.31 \pm 10.07$ & 0.31 \\
Ramipril & 34 & $3.27 \pm 1.71$ & & 11 & $4.09 \pm 1.26$ & 0.15 \\
Enalapril & 67 & $7.5 \pm 3.50$ & & 22 & $8.21 \pm 3.07$ & 0.50 \\
Hydrochlorothiazide & 35 & $14.82 \pm 5.26$ & & 10 & $18.25 \pm 6.24$ & 0.09 \\
\hline
\end{tabular}

Values are expressed as mean $\pm S D, n=$ number of patients because there was only a mild renal impairment (Crcl $<90 \mathrm{ml} / \mathrm{min}$ ) in the geriatric population.

There were no significant differences in the mean converted equivalent doses of beta-blockers, CCBs, ARBs, and hydrochlorothiazide when adjusted for the duration of hypertension and $\mathrm{Crcl}$ in geriatrics and non-geriatrics.

ACE inhibitors are widely used as preferred antihypertensive drugs for elderly patients with diabetes and nephropathy, due to their nephroprotective effect, low risk of hypotension, and metabolic neutrality. Several ACE inhibitors (benazepril, ramipril, and fosinopril) cleared by kidneys result in an increase in plasma concentration due to CKD or age-related renal changes [18]. The mean converted equivalent doses of ACE inhibitors were lower in geriatric population (7.2 mg) compared to non-geriatric population $(8.1 \mathrm{mg})$, although statistically insignificant this difference of $1 \mathrm{mg}$ /day may be clinically significant when we initiate therapy. In view of longer duration of hypertension and lower $\mathrm{Crcl}$, ACE inhibitor dose needs adjustment for geriatric population. Hence, doses were adjusted for the duration of hypertension and $\mathrm{Crcl}$ to comprehend the true impact of age on doses of ACE inhibitors. When doses of different ACE inhibitors are adjusted for the duration of hypertension of 6-7 years, a $13-15 \%$ reduction was found in geriatric population, and this too was statistically insignificant because of a small sample size. A 15-20\% dose reduction was noted in geriatric patients when adjusted for a $\mathrm{Crcl}$ of $65 \mathrm{ml} / \mathrm{min}$. When both the durations of hypertension and $\mathrm{Crcl}$ adjusted doses were considered, statistically and clinically significant dosage reductions of approximately $20 \%$ were noted among geriatric patients compared to non-geriatrics. This indicates that geriatric patients require an approximately 20\% lower dose of ACE inhibitors compared to their non-geriatric counterparts; this dose reduction is a function of age and is independent of renal function and duration of hypertension. This is important clinically because, at the study site, the protocol is to start with a lower dose of ACE inhibitors regardless of the age; at other centers where the ACE inhibitors are started at a higher dose, the dose reduction among geriatrics may be much more.

Most of the commonly used ACE inhibitors are prodrugs which undergo activation in the liver. Impaired biotransformation due to hepatic dysfunction, often found in the elderly, may require the dose to be increased [19]. However, in our study, we found the liver functions of the geriatric patients to be within the normal reference range. In our previous publication, the doses of sulfonylurea group of oral antidiabetic agents required in geriatrics were almost 20-25\% less compared to non-geriatrics as these drugs are mainly cleared through the kidney and also because the geriatric diabetic patients have concealed renal impairment compared to non-geriatrics [20]. The present study shows that only ACE inhibitors need reduction in drug dosage in geriatrics when adjusted for the duration of hypertension and $\mathrm{Crcl}$.

Table 5: Comparison of duration and Crcl adjusted dose of antihypertensive drugs in geriatrics and non-geriatrics

\begin{tabular}{|c|c|c|c|c|c|c|}
\hline \multirow[t]{2}{*}{ Drug, duration (years), $\mathrm{Crcl}(\mathrm{ml} / \mathrm{min})$} & \multicolumn{2}{|c|}{ Geriatrics } & \multicolumn{2}{|c|}{ Non-geriatrics } & \multirow[t]{2}{*}{$\mathbf{p}$} & \multirow[t]{2}{*}{ Dose: $\% \downarrow$} \\
\hline & $\mathbf{n}$ & Mean dose \pm SD (mg) & $\mathbf{n}$ & Mean dose \pm SD (mg) & & \\
\hline Beta-blockers, (8.62), (63.47) & 110 & $38.12 \pm 1.51$ & 39 & $39.25 \pm 2.68$ & 0.72 & No $\downarrow$ \\
\hline CCBs, (8.12), (62.95) & 90 & $4.96 \pm 0.27$ & 27 & $4.97 \pm 0.51$ & 0.95 & No $\downarrow$ \\
\hline ARBs, (9.37), (60.32) & 64 & $40.12 \pm 2.14$ & 20 & $36.61 \pm 4.24$ & 0.48 & No $\downarrow$ \\
\hline Hydrochlorothiazide, (8.96), (59.87) & 35 & $14.78 \pm 0.99$ & 10 & $18.86 \pm 1.97$ & 0.08 & No $\downarrow$ \\
\hline
\end{tabular}

Table 6: Comparison of duration and $\mathrm{Crcl}$ adjusted dose of ACEIs in geriatrics and non-geriatrics

\begin{tabular}{|c|c|c|c|c|c|c|}
\hline \multirow[t]{2}{*}{ Drug, duration (years) $\mathrm{Crcl}$ (ml/min) } & \multicolumn{2}{|c|}{ Geriatrics } & \multicolumn{2}{|c|}{ Non-geriatrics } & \multirow[t]{2}{*}{$\mathbf{p}$} & \multirow[t]{2}{*}{ Dose: $\% \downarrow$} \\
\hline & $\mathbf{n}$ & Mean dose \pm SD (mg) & $\mathbf{n}$ & Mean dose \pm SD (mg) & & \\
\hline Enalapril, (6.35), (64.75) & 67 & $7.17 \pm 0.42$ & 22 & $9.04 \pm 0.83$ & 0.06 & 20.57 \\
\hline Ramipril, (6.69), (64.54) & 34 & $3.29 \pm 0.29$ & 11 & $4.03 \pm 0.56$ & 0.27 & 18.36 \\
\hline ACEIs, (6.47), (64.68) & 101 & $7.02 \pm 0.34$ & 33 & $8.64 \pm 0.67$ & $0.04^{*}$ & 18.63 \\
\hline
\end{tabular}

${ }^{*} \mathrm{p}<0.05, \% \downarrow=$ percentage reduction 


\section{CONCLUSION}

Age does not influence drug dosage of antihypertensives except for ACE inhibitors. A 20\% dosage reduction is needed for ACEIs in the elderly, when adjusted for the duration of hypertension and Crcl.

\section{FUNDING STATEMENT}

Nil.

\section{ACKNOWLEDGMENT}

Nil.

\section{CONFLICTS OF INTEREST}

Nil.

\section{REFERENCES}

1. Joshi SR, Parikh RM. India-diabetes capital of the world: Now heading towards hypertension. J Assoc Physicians India 2007;55:323-4.

2. Dash SC, Agarwal SK. Incidence of chronic kidney disease in India. Nephrol Dial Transpl 2006;21:232-3.

3. Reboldi G, Gentile G, Angeli F, Verdecchia P. Choice of ACE inhibitor combinations in hypertensive patients with Type 2 diabetes: Update after recent clinical trials. Vasc Health Risk Manag 2009;5:411-27.

4. Franlin SS, Jacobs MJ, Wong ND, L'Italian GL, Lapuerta P. Predominance of isolated systolic hypertension among middle age and elderly US hypertensives analysis based on national health and nutrition examination survey (NHANES)III. Hypertension 2001;37:869-74.

5. Sowers JR, Epstein M, Frohlich ED. Diabetes, hypertension and cardiovascular disease; An update. Hypertension 2001;37:1053-9.

6. Keller CK, Bergis KH, Fliser D, Ritz E. Renal findings in patients with short term Type 2 diabetes. J Am Soc Nephrol 1996;7:2627-35.

7. Adler AI, Stratton MI, Neil AW, Yudkin JS, Matthews DR, Cull AC, et al. Association of systolic blood pressure with macrovascular and microvascular complications of Type 2 diabetes (UKPDS36):
Prospective observational study. BMJ 2000;321:412-9.

8. Wasen E, Isoaho R, Mattila K, Vahlberg T, Kivela SL, Irjala K. Renal impairment associated with diabetes in the elderly. Diabetes Care 2004:27:2648-53

9. Odegard PS, Setter SM, Neumiller JJ. Considerations for the pharmacological treatment of diabetes in older adults. Diabetes Spectrum 2007;20:239-47.

10. Abaterusso C, Lupo A, Ortalda V, De Biase V, Pani A, Muggeo M, et al. Treating elderly people with diabetes and stage 3 and 4 chronic kidney disease. Clin J Am Soc Nephrol 2008;3:1185-94.

11. Available from: http://www.med.unc.edu/im/files/internal-medicineclinic-documents/HTN_NONDM 7.06 V5.pdf.

12. Weber MA. Outcomes of treating hypertension in the elderly: A short commentary on current issues. Am J Geriatr Cardiol 2003;12:14-8.

13. Hernandez-Vila E. A review of the JNC 8 blood pressure guideline. Tex Heart Inst J 2015;42:226-8.

14. Altaf M, Rasheed A, Mujtaba A, Mohammed S. Drug utilization evaluation of antihypertensives in geriatric patients in a tertiary care hospital. Int J Pharm Pharm Sci 2014;6:261-4.

15. Borzecki AM, Glickman ME, Kader B, Berlowitz DR. The effect of age on hypertension control and management. Am J Hypertens 2006; 19:520-7.

16. Chowta MN, Adhikari PM, Raj S, Laxman M, Kariappa A, George J, et al. Evaluation of appropriateness of prescription and polypharmacy in the geriatric population: A cross-sectional study at a comprehensive geriatric clinic in a tertiary care hospital. Int J Pharm Pharm Sci 2016;8:119-23.

17. Carter BL. Dosing of antihypertensive medications in patients with renal insufficiency. J Clin Pharm 1995;35:81-6.

18. Mangoni AA, Jackson HD. Age related changes in pharmacokinetics and pharmacodynamics: Basic principles and practical applications. $\mathrm{Br}$ J Clin Pharm 2004;57:6-14.

19. Dickerson LM, Gibson MV. Management of hypertension in older persons. Am Fam Physician 2005;71:469-76.

20. Shastry R, Adhikari P, Kamath A, Chowta M, Ullal S, Pai M. Do geriatrics require dose titration for antidiabetic agents? J Postgrad Med 2013;59:271-4. 